\title{
97. Discharge Process in Geiger-Müller Counters. IX.
}

Dead Time.

\author{
By Osamu Minakawa. \\ Institute of Physical and Chemical Research, Tokyo. \\ (Comm. by S. Nishikawa, M.I.A., July 12, 1944.)
}

Generally the size of the individual pulse of a G. M. counter, at a constant over-voltage is almost equal despite the amount of the primary ionization. When, however, the counting rate is very large, and the time interval between the two successive pulses is very small, the size of the pulse of the latter discharge is more or less smaller than the normal value, and the shorter the interval, the smaller the pulse size. In the limiting case the pulse size becomes zero; in other words, within a certain time interval after the discharge, the counter does not give any measurable pulse even when the ionizing particle enters into the counting volume. This time interval is called the dead time of a counter. These circumstances can clearly be observed on an oscillograph screen by the use of a single sweep time axis. Examples of the photos are shown in Fig. 1.

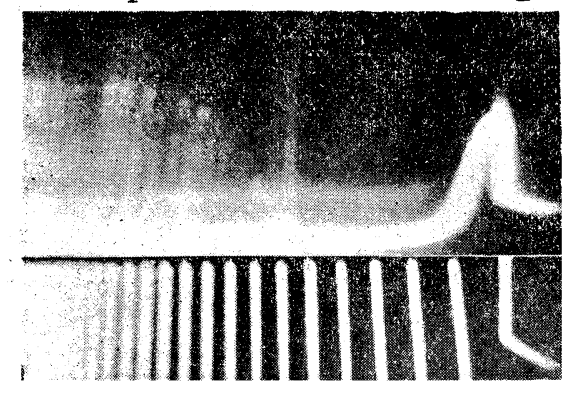

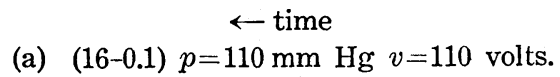

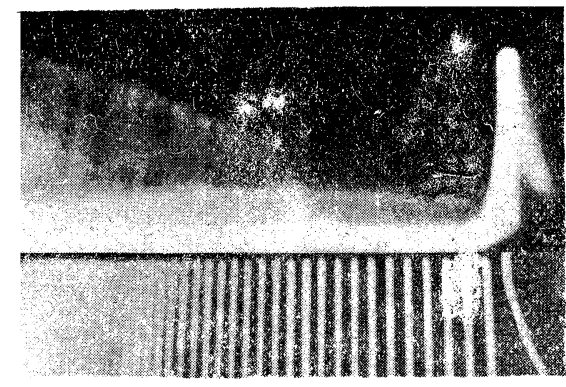

(b) $(45-0.1) p=110 \mathrm{~mm} \mathrm{Hg} v-500$ volts.

Fig. 1.

The lower part of the figures gives the time scale using relaxation oscillator $\left(\omega=5 \times 10^{4}\right)$.

It has already been reported ${ }^{1)}$ that the dead time depends on the applied voltage, the geometrical construction, and the pressure of filling gas and vapour. These can be explained by the shielding action of positive ion sheath as described below.

The positive ion sheath produced in a discharge moves in the form of a cylinder towards the cathode wall owing to the electric fields which are present in the counter.

If the distributed capacity of the central wire system is large, it can be considered that the variation of potential of the wire is negligible even when all electrons were collected to the wire and the posi-

1) O. Minakawa, Proc. 19 (1943), 642, 20 (1944), 77. 
tive ions moved appreciably. Let $Q_{V}$ be the charge in the counter capacity per unit length, when negative high voltage $-V$ is applied to the counter wall. If we assume that there is a positive space charge sheath of negligible thickness, for simplicity, at the distance $r$ from the axis of the wire with charge $q$ per unit length, and that the charge on the wire turns out to be $Q(r)$, then it is given by

$$
Q(r)=Q_{V}-2 c q \log \frac{r_{a}}{r}, \text { where } c=\frac{1}{2 \log \frac{r_{a}}{r_{i}}},
$$

where $r_{a}$ and $r_{i}$ are the radii of wall and wire of the counter, respectively.

It can be considered that the electric field in the vicinity of the wire surface at the end of the dead time is equal to that of the threshold value, viz. $Q=c V_{t}, V_{t}$ being the threshold voltage. Accordingly we obtain

$$
V-V_{t}=v=2 q \log \frac{r_{a}}{r_{\tau}}
$$

where $r_{\tau}$ is the radius of the positive ion sheath at the end of the dead time. From the measurements of charge $q$ with over voltage $v$, we can find $r_{r}$.

As to the motion of the positive ions, if we assume their mobility to be constant ${ }^{*}$ in a first approximation, then

$$
\frac{d r}{d t}=k \frac{760}{p} E(r),
$$

where $p$ is the value of the pressure in $\mathrm{mm} \mathrm{Hg}$ and $E(r)$ is the electric field strength. Just out of the positive ion sheath $E(r)$ is given by

$$
E(r)=\frac{2(Q+q)}{r}=\frac{2\left\{Q_{V}+q\left(1-\frac{\log \frac{r_{a}}{r}}{\log \frac{r_{a}}{r_{i}}}\right)\right\}}{r} .
$$

Putting this expression into (3) and integrating, we obtain the following relation.

$$
2 k \frac{760}{p} \tau=\int_{r_{i}}^{r_{\tau}} \frac{r}{\left\{Q_{V}+q\left(1-\frac{\log \frac{r_{a}}{r}}{\log \frac{r_{a}}{r_{i}}}\right)\right\}} d r
$$

*) The mobility of positive ions in the gas mixture (alcohol and argon) and in the extremely high field which are present in a counter, are not yet known completely. It may not be a constant, but may depend upon the field strength in a complicated manner. 
The right hand side of the above equation is not integrable in a simple form, and accordingly it was evaluated numerically, and the value of $k$ was obtained from the experimental data. The results of the calculations are shown in Table I. It is rather surprising that the values of $k$ obtained with various conditions are almost equal and are

TABLE I. $\left(\mathrm{C}_{2} \mathrm{H}_{5} \mathrm{OH}: A=1: 10\right)$.

\begin{tabular}{|c|c|c|c|c|c|c|c|c|}
\hline $\begin{array}{c}r_{i} \\
(\mathrm{~cm})\end{array}$ & $\begin{array}{c}r_{a} \\
(\mathrm{~cm})\end{array}$ & 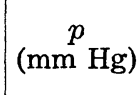 & $\begin{array}{c}v \\
\text { (volts) }\end{array}$ & $\begin{array}{c}V_{t} \\
\text { (volts) }\end{array}$ & $\begin{array}{c}q \\
\left(10^{-10}\right. \\
\text { coulomb) }\end{array}$ & $\left(10^{-\tau^{\tau}} \mathrm{Sec}\right)$ & $\begin{array}{c}r_{\tau} \\
(\mathrm{cm})\end{array}$ & $k$ \\
\hline 0.0058 & 0.8 & 110 & 108 & 780 & 1.01 & 9.5 & 0.44 & 0.46 \\
\hline ", & " & ", & 195 & $"$ & 1.58 & 6.2 & 0.40 & 0.46 \\
\hline " & " & $"$ & 282 & $"$ & 2.11 & 4.3 & 0.38 & 0.45 \\
\hline 0.0105 & $"$ & , & 108 & 860 & 1.23 & 9.0 & 0.49 & 0.47 \\
\hline 0.0165 & " & $"$ & 108 & 940 & 1.54 & 8.6 & 0.57 & 0.56 \\
\hline 0.0058 & 1.5 & ", & 113 & 900 & 0.67 & 24 & 0.59 & 0.39 \\
\hline " & 2.25 & $"$ & 118 & 990 & 0.59 & 36 & 0.73 & 0.41 \\
\hline 0.0165 & ", & " & 118 & 1240 & 0.72 & 44 & 0.91 & 0.53 \\
\hline 0.0058 & 0.8 & 165 & 108 & 930 & 0.96 & 11.5 & 0.43 & 0.45 \\
\hline
\end{tabular}

numerically of the right order, considering the ions produced are almost alcohol ions. ${ }^{1)}$ In other words the dead time of counters in various conditions are approximately calculable from the value of $k$. But in reality the positive ion sheath has some thickness and the field strength through the thickness is not constant so that the broadening of thickness may take place. Accordingly when the over-voltage is

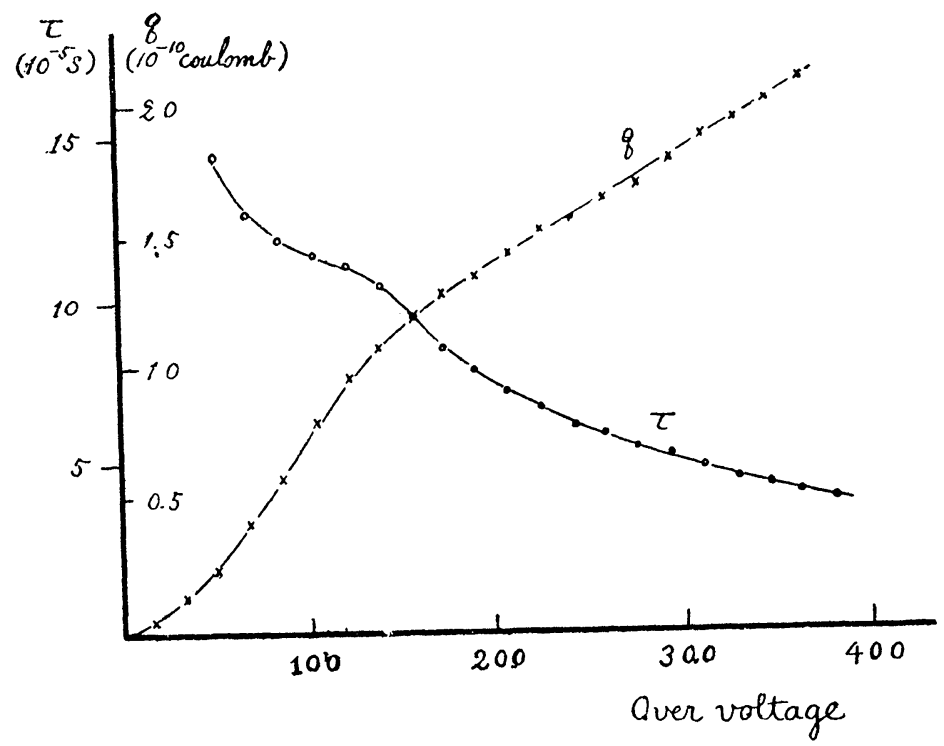

Fig. 2. $(16-0.1) p=165 \mathrm{~mm} \mathrm{Hg}$.

1) O. Minakawa, Proc. 19 (1943), 19. 
high, i. e. when $q$ becomes large, the results of the above calculations deviate in the direction of under estimation of $k$.

At any rate, the existence of dead time and the dependence of pulse size on the time interval after the occurrence of the preceding pulse can be understood by the shielding action of positive ion sheath. The dependence of dead time upon $v$ or $q$ will clearly be seen from Fig. (2).

In conclusion the writer wishes to express his cordial thanks to Prof. H. Nagaoka and to Prof. S. Nishikawa for their interests throughout this work. The cooperation and assistance of Miss T. Kamada are highly appreciated. 